in biopsy samples of eight testes from six of 555 infertile men. ${ }^{5}$ Four of the six patients developed an invasive tumour between one and five years after the first biopsy; no tumours were reported in the remainder. Similar results have been published by a group in Switzerland, who found abnormalities in nine of 1635 infertile men, of whom five developed malignant tumours within six years. ${ }^{6}$ This is powerful evidence that the atypical appearances of the germ cells are premalignant, and the condition may reasonably be classified as carcinoma in situ. Presumably the abnormality is sufficiently widespread in the testis to be detected on a random biopsy. More circumstantial evidence for the hypothesis was given in a histological study of the macroscopically normal tissue surrounding germ-cell tumours. In 17 of 22 such testes widespread foci of carcinoma in situ were found. ${ }^{7}$ Examples of both seminoma and teratoma were found to coexist with such atypical cells, and the hypothesis has now been extended to propose that both types of tumour can develop from the same premalignant cell-a proposition in accordance with the theory of their common germ-cell origin. ${ }^{8}$

The hypothesis that carcinoma in situ precedes invasive germinal cell neoplasms seems, then, to be proved in infertile men. Other work suggests that it may be of more general importance in explaining the action of factors known to predispose to development of tumours of the testis. The risk that a tumour will develop in a cryptorchid testis is usually put at about 30 times that in a normally located testis. ${ }^{910}$ This extra risk is thought to affect the testis even after orchidopexy, though the recent trend towards fixation in early childhood may reduce the adverse chances. Atypical germinal cells have occasionally been described in cryptorchid testes, ${ }^{11} 12$ but Skakkebaek et al ${ }^{13}$ discovered carcinoma in situ in four out of 50 men treated previously for maldescended testis. Two of these patients had an unsuspected invasive tumour in adjacent testicular tissue. In all four patients the contralateral testis was normal, whereas the affected testis was small and atrophic in three cases. Possibly either a developmental anomaly or an acquired defect in growth (attributable to the enforced residence of the testis in a hostile environment) predisposes it to dysplastic tubular growth and so to neoplasia.

One more example of the association between carcinoma in situ and tumour growth in an abnormal gonad is found in the testicular feminisation syndrome. ${ }^{14}$ Of greater importance, however, is the known tendency for a second germ-cell tumour to develop in the contralateral testis of men who have already had a testicular tumour. ${ }^{9}$ Once again Skakkebaek's work has shown a correlation between carcinoma in situ and tumour development. Four of 50 patients who had had orchidectomy for a tumour showed carcinoma in situ, and one patient showed definite signs of invasive tumour on serial biopsy four years later. ${ }^{15}$

There is no doubt now that carcinoma in situ is a premalignant change and, indeed, analysis of data on 2000 or so infertile patients has suggested that the 18 testes with this tubular abnormality had a $70 \%$ chance of developing a tumour within five years of the first positive biopsy sample. ${ }^{16}$ Clearly a substantial proportion of testicular neoplasms could be prevented by orchidectomy alone at a premalignant stage. The problem lies in selection of patients at risk, since once a patient has been subjected to a biopsy the test should, logically, be repeated at regular intervals. Skakkebaek' ${ }^{5}$ advice is that testicular biopsy may be useful for the early detection and cure of germ-cell carcinoma in patients with cryptorchidism, infertility, or previous cancer in one testis. In practice, in infertile men biopsy may reasonably be limited to men with small testes. More uncertain, however, is the policy for patients who have undergone orchidopexy for undescended testes; the evidence so far suggests that serial biopsy would be fully justified. The patient who has had a testicular tumour presents a more difficult problem, since many urologists would shrink from recommending surgical removal of a young man's remaining testis on the basis of a few atypical cells: some might not want to risk finding them by doing a biopsy at all.

Finally, what are the risks in taking an open scrotal biopsy specimen from a potentially malignant testis ${ }^{17}$ Reassuringly, there is no evidence at present that an open biopsy of a testis with carcinoma in situ could lead to scrotal metastasis.

1 Anon. An epidemic of testicular cancer? Lancet 1968;ii:164-5.

2 Peckham MJ, McElwain TJ, Barrett A, Hendry WF. Combined management of malignant teratoma of the testis. Lancet 1979;ii:267-70.

${ }^{3}$ Skakkebaek NE. Abnormal morphology of germ cells in two infertile men. Acta Pathol Microbiol Scand 1972;80A:374-8.

${ }^{4}$ Skakkebaek NE. Possible carcinoma-in-situ of the testis. Lancet 1972; ii:516-7.

${ }^{5}$ Skakkebaek NE. Carcinoma in situ of the testis : frequency and relationship to invasive germ cell tumours in infertile men. Histopathology $1978 ; 2: 157-70$.

${ }^{6}$ Nuesch-Bachmann IH, Hedinger C. Atypische spermatogonien als präkanzerosc. Schwei. Med Wochenschr 1977;107:795-801.

"Skakkebaek NE. Atypical germ cells in the adjacent "normal" tissue of testicular tumours. Acta Patho Microbiol Scand 1975;83A :127-30.

${ }^{8}$ Collins DH, Pugh RCB. Classification and frequency of testicular tumours. Br $\mathcal{F}$ Urol 1964;36,suppl:1-11.

${ }^{9}$ Gilbert JB, Hamilton JB. Studies in malignant testis tumours. IIIIncidence and nature of tumours in ectopic testes. Surg Gynecol Obstet $1940 ; 71: 731-43$.

10 Whitaker RH. Congenital disorders of the testicle. In Blandy JP, ed. Urology. Oxford: Blackwell, 1976;1153-78.

11 Waxman M. Malignant germ cell tumor in situ in a cryptochid testis. Cancer 1976;38:1452-6.

12 Williams TR, Brendler H. Carcinoma in situ of the ectopic testis. $\mathcal{F}$ Urol 1976;117:610-2.

${ }^{13}$ Krabbe S, Skakkebaek NE, Berthelsen JG, et al. High incidence of undetected neoplasia in maldescended testes. Lancet 1979; ;:999-1000.

14 Skakkebaek NE. Carcinoma-in-situ of testis in testicular feminization syndrome. Acta Pathol Microbiol Scand 1979;87A :87-9.

${ }^{15}$ Berthelsen JG, Skakkebaek NE, Mogensen P, Sorensen BL. Incidence of carcinoma in situ of germ cells in contralateral testis of men with testicular tumours. $B r \operatorname{Med} \mathcal{F} 1979$;ii :363-4.

${ }^{16}$ Skakkebaek NE, Berthelsen JG. Carcinoma-in-situ of testis and orchiectomy. Lancet 1978; ;i:204-5.

17 Blandy JP, Hope-Stone HF, Dayan AD. Tumours of the testicle. London: Heinemann, 1970.

\section{Opinion polls and kidney transplantation}

Opinion polls provide a reliable means of testing public reactions, and several have been commissioned on attitudes to kidney transplantation. A poll commissioned by the DHSS and carried out by Marplan Ltd in 1978 questioned 1411 people, about carrying donor cards, public donation, and the possibilities of an opting-out system (kidneys to be donated unless a specific injunction to do so has been registered by the donor), or opting-in (kidneys to be used only if a specific approval has been given before the event and thus registered by the prospective donor). The results were very similar to those of a National Opinion Polls Random Omnibus Survey carried out earlier in 1978, and to a Gallup Poll commissioned privately in 1979 on behalf of the Wessex Regional Transplant Unit at St Mary's Hospital, Portsmouth. Respectively the percentages of the sample carrying donor cards in the 1979 and the two 1978 polls were $12 \%, 10 \%$, and $8 \%$. As a reflection on the frailties of human nature, however, the 1978 poll showed that, even though $12 \%$ of the sample had obtained a donor card 
and filled it in, only $9 \%$ had filled it in properly and only 5\% were actually carrying it on their person at the time of the interview.

The small numbers of persons who obtain and fill in a donor card and are effective card carriers contrast vividly with the proportion of the population who say that they. support kidney donation and who would themselves donate a kidney in the event of their death. These figures were in the 1979 poll $48 \%$ and in the 1978 polls $57 \%$ and $69 \%$. Furthermore, only $20 \%, 21 \%$, and $18 \%$ respectively were actively opposed to kidney donation. So while only a small minority of people are effective card carriers, an overwhelming four to one majority of the population is in agreement with the principle of kidney donation. Probably the reason for this disparity is inertia. All three surveys found that people were well informed about the treatment of end-stage kidney failure by transplantation or dialysis; indeed, the Marplan survey suggested that public attitudes would not be appreciably changed by the wider dissemination of facts and figures about the needs and benefits of kidney transplantation. On the other hand, a small pilot study arranged as a follow-up to the Portsmouth survey suggested that dissemination of information had a sixfold effect in changing public attitudes to the carrying of donor cards. The dissemination of information seems likely to be the single most powerful factor in obtaining public approval for kidney transplantation. In all three surveys, there was a clear majority $(75 \%, 69 \%, 58 \%$ respectively) who were entirely opposed to the concept of "opting-out," reflecting public anxiety about giving the State rights over the individual, even after death. Experience in France with an opting-out system suggests that it is not very effective in practice. The donation of kidneys in France has not increased substantially since the opting-out legislation was passed, nor has it been happily accepted by the population.

What we require is a practical and effective system whereby those people who have decided that there is a social obligation to donate their kidneys after death can register that fact before the event, with provision for rapid ascertainment of that decision at or around the time of death. Such a system would avoid the need for painful decisions to be made by the next of kin at a highly inopportune time.

\section{Bronchiolitis in infancy and childhood}

The anatomical classification of acute respiratory tract infection in infants and children into upper (cold, pharyngitis, otitis), middle ("croup," epiglottitis), and lower (bronchitis, bronchiolitis, pneumonia) is useful clinically, ${ }^{1}$ despite the limitations imposed by its arbitrarily defined boundaries. Having made this first-stage diagnosis, the family doctor next assesses clinical severity, the need for any immediate treatment, and whether referral to hospital is indicated. Finally, when he also takes into account the age of the child, season of the year, and concurrent epidemics of respiratory illness, he may often be able to decide on an aetiological diagnosis. During the winter months the most likely cause of a respiratory infection in infants is the respiratory syncytial virus. ${ }^{2}$

The most common lower respiratory tract infection in infancy is acute bronchiolitis, usually defined as an acute respiratory infection affecting children aged under 1 year in which coryzal symptoms precede cough, tachypnoea, and sometimes expiratory wheezing due to inflammatory obstruction of the small airways of the lung. Physical signs include overinflation of the chest, symmetrical retractions, and widespread fine crepitations, and sometimes also expiratory rhonchi, on auscultation. Epidemics occur each winter in Britain and elsewhere. The main virus responsible is respiratory syncytial virus, which can be isolated or detected by immunofluorescence studies of nasopharyngeal secretions in over $70 \%$ of cases. $^{2}$ Other agents include parainfluenza, adenovirus, and Mycoplasma pneumoniae. Pathological studies ${ }^{3}$ of the lungs of infants dying of bronchiolitis show necrosis of epithelial cells in peripheral airways, proliferation of mucosal cells, and lymphocytic infiltration of peribronchiolar tissues.

The clinical definition "acute bronchiolitis" has, however, been criticised as encouraging misconceptions about the prevalence and course of histopathological "bronchiolitis." Inflammatory obstruction of the small airways might be the pathological denominator in a wider range of respiratory infections associated with wheeze, including not only acute bronchiolitis in infancy but also recurrent wheezy bronchitis in young children and virus-induced bronchospasm in atopic children of all ages. The differing clinical manifestations of these conditions would then be explicable solely on the basis that in young children the peripheral airways resistance is relatively higher ${ }^{4}$ than in older children and adults, so increasing their susceptibility to obstruction of the peripheral airways in response to infection. Certainly we have no clear explanation of why acute bronchiolitis in infancy tends to be most severe in the narrow age span between 1 and 6 months.

A recent epidemiological study ${ }^{5}$ of respiratory illnesses associated with wheeze in children has attempted to broaden the traditional clinical concept of bronchiolitis and from a community in the United States has provided new information on the aetiology and precipitants of wheezing in children. In children studied during 11 years wheeze was the main symptom in 1851 of 6165 episodes of lower respiratory illnesses. About half of wheezy children were under the age of 2. Non-bacterial pathogens were isolated from $21 \%$ of the wheezy children, with respiratory syncytial virus, parainfluenza 1 and 3, adenovirus, and Mycoplasma pneumoniae accounting for $81 \%$ of the isolates. Respiratory syncytial virus was the most common precipitant of wheeze under the age of 5 and $M$ pneumoniae the main agent in children of school age. The epidemiological evidence presented suggests that even when isolates were not obtained wheezing was due to an infective illness.

The American authors recommended that the term "wheeze-associated respiratory infections" should be adopted by clinicians to describe the broad range of illnesses in which infection and wheeze are associated. Their suggestion could, however, exaggerate rather than resolve existing difficulties: their assumptions about the range of "bronchiolitis" are unproved; they failed to define "wheeze"-a symptom not invariably present in bronchiolitis-and did not consider the atopic state of children included in their study. The conventional, albeit imperfect, clinical terminology should be retained for the present while we plan fresh approaches to unravel the interactions of infection, allergic state, and wheezing tendency and their relation to episodic or persistent peripheral airways obstruction during childhood.

1 Court SDM. The definition of acute respiratory illness in children Postgrad Med f 1973;49:771-6.

2 Report to the Medical Research Council Subcommittee on Respiratory Syncytial Virus Vaccines. Respiratory syncytial virus infection: admissions to hospital in industrial, urban, and rural areas. $\mathrm{Br} \mathrm{Med} \mathscr{f}$ 1978 ;ii :796-8. 